\title{
Optimal cooling of a driven artificial atom in dissipative environment
}

\author{
Lingjie Du, Yang Yu, and Dong Lan \\ National Laboratory of Solid State Microstructures and Department of Physics \\ Nanjing University, Nanjing 210093, China \\ E-mail: Lingjie.Du@rice.edu \\ yuyang@nju.edu.cn \\ landong89@gmail.com
}

Received July 30, 2012

\begin{abstract}
We study microwave-driven cooling in a superconducting flux qubit subjected to environment noises. For the weak decoherence, our analytical results agree well with the experimental observations and show that the microwave amplitude for optimal cooling should depend linearly on the dc flux detuning. With the decoherence stronger, more vibrational degrees of freedom (analogous with atomic physics) couple in, making the ordinary cooling method less effective or even fail. We propose an improved cooling method, which can eliminate the perturbation of additional vibrational degrees of freedom hence keep high efficiency, even under the strong decoherence. Furthermore, we point out that the decoherence can tune the frequency where microwave-driven Landau-Zener transition reaches maximum, displaying the feature of incoherent dynamics which is important for the optimal cooling of qubits and other quantum systems.
\end{abstract}

PACS: 37.10.De Atom cooling methods;

03.65.Yz Decoherence; open systems; quantum statistical methods;

85.25.-j Superconducting devices;

03.67.Lx Quantum computation architectures and implementations.

Keywords: superconducting devises, qubit, microwave-driven cooling, decoherence.

\section{Introduction}

Superconducting devises based on Josephson tunnel junctions, shown to act as artificial atoms, can be used to demonstrate quantum phenomena at macroscopic scales and hold promise for applications in quantum computation as qubits [1-6]. Qubits are very delicate and their manipulation with electronics needs to be isolated from the disturbance of the environmental thermal noise [7-21]. Therefore, cooling the qubits, considered as a straightforward means to preserve the coherence of qubits, paves the way for remarkable achievements in demonstrating various quantum coherent phenomena. In general, the dilution refrigeration is used for cooling with intrinsic drawbacks such as limited cooling efficiency and poor heat conduction. Therefore, developing novel cooling techniques is crucial to improve the performance of qubits.

Recently an interesting technique, microwave-driven cooling, was developed from the extensive investigations on coupled systems of the qubits and quantum resonators. It was found that the qubit can cool down the resonator [22-25]. On the other hand, the inverse process, using a "resonator" to cool a qubit, was also demonstrated experimentally by Valenzuela et al. [26] in a superconducting flux qubit [27,28]. Driving the system with microwave of the amplitude $\Phi_{r f}=\Phi_{12}\left[\Phi_{12}\right.$ is defined in Fig. 1(a) $]$ and the frequency $5 \mathrm{MHz}$, they reduced the effective temperature of the qubit to $3 \mathrm{mK}$, which was two orders of magnitude lower than the surrounding environment temperature. This process is analogous to the optical sideband cooling [29-32], where a twolevel system (TLS) in an ion (a qubit) is used to cool ion trap potential (an oscillator), subsequently providing direct cooling to the degrees of freedom of interest. But in contrast to the optical method, here an ancillary oscillator-like state is coupled to a qubit by a side crossover, and cools the qubit through microwave-driven Landau-Zener (MDLZ) transitions [or Landau-Zener-Stückelberg (LZS) interferometry] $[18,33,34]$. As the qubit is driven through a degeneracy point (or crossover), Landau-Zener (LZ) transition happens [35], which usually is used to enhance the quantum tunneling rate, [36,37], to prepare the quantum states [38], and to control the gate operations [39]. When a periodic strong driving is applied to the qubit, the qubit undergoes repeated LZ transitions 
at the degeneracy point. If the driving frequency is larger than the decoherence rate, the repetition of $\mathrm{LZ}$ transitions can induce quantum interference, which leads to an oscillatory dependence of qubit population in the final state on the detuning from the degeneracy point and the microwave amplitude, known as LZS interferometry [18-20,26,40-50].

In this paper, we study the optimal cooling conditions based on a microscopic model of LZS interferometry in the multi-level system [49]. For the situation of Ref. 25, we found that the microwave amplitude for optimal cooling should depend linearly on the dc flux detuning. Then we demonstrate that strong decoherence might couple the qubit to another oscillator-like state. The coupled system can interact with the original oscillator-like state, destroying the cooling efficiency in the ordinary method. In order to realize the cooling under the strong decoherence, we propose an exquisite microwave manipulation to exclude the excessive oscillator-like state. Furthermore, in the previous work, the effective cooling is realized by choosing a constant extremely low microwave frequency $(5 \mathrm{MHz})$. However, we found that the frequency for optimal cooling relies on the decoherence. The decoherence will tune the frequency corresponding to the maximum rate of MDLZ transition, leading to the change of the frequency condition in both methods. In the strong decoherence environment, LZS interference displays the stationary population of incoherent evolution. While the trend of quantum information is coherent manipulation, our analysis shows the importance of the incoherent LZS dynamics in quantum cooling. In addition, active cooling of qubits provides an effective means for qubit state preparation and for suppressing the decoherence in multi-qubit systems, even under the strong decoherence.

The organization of this paper is as follows. In Sec. 2, we give the basic model of LZS interferometry and the principle of microwave cooling. In Sec. 3, we present a detailed analysis of the amplitude conditions for optimal cooling under the weak decoherence with the ordinary cooling method. In Sec. 4, we study the cooling under the strong decoherence. An improved cooling method is proposed. In Sec. 5, we study the frequency conditions for optimal cooling.

\section{Basic model}

We focus on a multi-level superconducting quantum device, which consists of a superconducting loop interrupted by three Josephson junctions $[18,26,42]$. If the external flux bias $\Phi \approx 0.5 \Phi_{0}$, where $\Phi_{0}=h / 2 e$ is the flux quantum, a double well landscape (Fig. 1) of the potential energy parameterized by the dc flux detuning $\delta \Phi_{d c}=\Phi-0.5 \Phi_{0}$ exists in the system. At the millikelvin temperature $\sim 10 \mathrm{mK}$, a series of diabatic states, denoted as $|i\rangle$ and $|j\rangle$, respectively, are localized in different wells with the crossovers $\Delta_{i j}$ between states $|i\rangle$ and $|j\rangle$ (i=0, 2, right well with negative slope; $j=1$, 3 , left well with positive slope). We assume
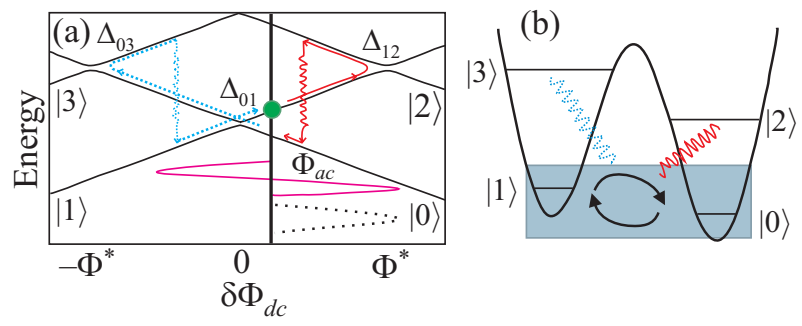

Fig. 1. (Color online) (a) Schematic energy diagram of multilevel superconducting quantum system. Red solid curve represents the microwave $\Phi_{a c}$ in the ordinary cooling method in Sec. 3. Black dashed curve represents the microwave in the improved cooling method in Sec. 4. The thick vertical black line marks a particular static flux detuning $\delta \Phi_{d c}$. States $|1\rangle$ and $|3\rangle$ are in the left well; $|0\rangle$ and $|2\rangle$ are in the right well. The red solid path describes the cooling with the population in state $|1\rangle$ transferred to $|0\rangle$. The blue dot path represents the population transferred from state $|0\rangle$ to $|1\rangle$. (b) Schematic picture of double-well potential of the system. The shaded region defines the qubit. Conventionally, we employ a microwave to couple a oscillator-like state $|2\rangle$ to the qubit. However, the strong decoherence would make another oscillator-like state $|3\rangle$ destroys the cooling completely, although state $|3\rangle$ has little effect under the weak decoherence.

the barrier is relatively high thus the tunneling strength $\left(\Delta_{i j}\right)$ is small. Two wells correspond to clockwise and anticlockwise flowing directions of the persistent currents, respectively. $\Phi^{*}\left(-\Phi^{*}\right)$ is the flux detuning at which the crossover $\Delta_{12}\left(\Delta_{03}\right)$ is reached. As the system is driven with a microwave $\Phi_{a c}=\Phi_{r f} \sin \omega t$, where $\Phi_{r f}$ is the microwave amplitude in the unit of magnetic flux, the time dependent flux detuning is

$$
\delta \Phi(t)=\delta \Phi_{d c}+\Phi_{a c} .
$$

Then the time dependent energy of eigenstate $|i\rangle$ can be described as

$$
\varepsilon_{i}(t)=\varepsilon_{i}^{*} \pm m_{i}\left(\delta \Phi_{d c}+\Phi_{r f} \sin \omega t\right),
$$

where $\varepsilon_{i}^{*}$ is the energy spacing between state $|i\rangle$ and the lowest state in the same well at $\delta \Phi(t)=0 \quad \mathrm{~m} \Phi_{0}$. $\varepsilon_{i}=\varepsilon_{i}^{*} \pm m_{i} \delta \Phi_{d c}$ is the dc energy of eigenstate $|i\rangle$, , " + " corresponds the states in the left well, "-" corresponds the states in the right well), and $m_{i}=d E_{i}(\Phi) / d \Phi$ is the energy level slope of $|i\rangle$.

When the state $|i\rangle$ is driven through the crossover $\Delta_{i j}$ at the time $t=t_{0}$, the probability staying at state $|i\rangle$ after $\mathrm{LZ}$ transition is given by (we set $\hbar=k_{B}=1$ )

$$
p=\exp \left(-\frac{\Delta_{i j}^{2}}{4 \zeta}\right),
$$


where the relative-energy sweep rate

$$
\zeta=\left.\frac{d}{d t}\left[\left|\varepsilon_{i}(t)-\varepsilon_{j}(t)\right|\right]\right|_{t=t_{0}} .
$$

For the case of the sinusoidal driving, we have

$$
\left|\varepsilon_{i}(t)-\varepsilon_{j}(t)\right|=\left(\left|m_{i}\right|+\left|m_{j}\right|\right) \Phi_{r f} \sin \omega t
$$

and

$$
\zeta=\left(\left|m_{i}\right|+\left|m_{j}\right|\right) \omega \Phi_{r f}\left|\cos \omega t_{0}\right|
$$

Therefore $\zeta \propto \omega$, which means that the larger frequency leads to the larger probability of LZ transition.

To describe the long-time evolution of driven multilevel quantum system interacting with the dissipative environment, we employ the rate equation approach 51]. In this case, MDLZ transition between two states in opposite wells needs to be considered. We use the Gaussian white noise model and perturbation theory $[47,49,52]$ to derive MDLZ transition rate from state $|i\rangle$ to $|j\rangle$ as

$$
W_{i j}=\frac{\Delta_{i j}^{2}}{2} \sum_{n} \frac{\left(\Gamma_{2}+\gamma\right) J_{n}^{2}\left(A_{i j} / \omega\right)}{\left(\varepsilon_{i j}-n \omega\right)^{2}+\left(\Gamma_{2}+\gamma\right)^{2}},
$$

where $\varepsilon_{i j}=\varepsilon_{i}-\varepsilon_{j}$ is the dc energy detuning of states $|j\rangle$ and $|i\rangle, \quad A_{i j}=\left|m_{i}\right| \Phi_{r f}+\left|m_{j}\right| \Phi_{r f}$ is the energy amplitude of the microwave, $\Gamma_{2}$ is the dephasing rate, $\gamma=$ $=\left(\Gamma_{j 1}+\Gamma_{i 0}\right) / 2, \Gamma_{j 1}$ and $\Gamma_{i 0}$ are the intrawell relaxation rate from states $|j\rangle$ and $|i\rangle$ in the left and right well, respectively. Of course, for $i=0$ or $j=1$, the correspondding $\Gamma_{i 0}$ or $\Gamma_{j 1}$ is zero. The term $\Gamma_{2}+\gamma$ describes the decoherence in this subsystem composed of states $|j\rangle$ and $|i\rangle$. In this article, when considering different decoherence, we will keep the intrawell relaxation rate $\Gamma_{31}=\Gamma_{20}=2 \pi \times 0.1 \mathrm{GHz}$ constant [49], changing only the dephasing rate. In order to analyze the optimal cooling, we consider four lowest states, and the qubit state occupations $p_{i}(i=0,1,2,3)$ follow $[53,54]$ :

$$
\begin{gathered}
\dot{p}_{00}=-\left(\Gamma_{01}^{\prime}+W_{03}+W_{01}\right) p_{00}+\left(\Gamma_{10}^{\prime}+W_{10}\right) p_{11}+ \\
+W_{30} p_{33}+\Gamma_{20} p_{22}, \\
\dot{p}_{11}=-\left(\Gamma_{10}^{\prime}+W_{12}+W_{10}\right) p_{11}+\left(\Gamma_{01}^{\prime}+W_{01}\right) p_{00}+ \\
+\Gamma_{31} p_{33}+W_{21} p_{22}, \\
\dot{p}_{22}=-\left(\Gamma_{20}+W_{21}+W_{23}\right) p_{22}+W_{32} p_{33}+W_{12} p_{11}, \\
p_{00}+p_{11}+p_{22}+p_{33}=1,
\end{gathered}
$$

where $\Gamma_{10}^{\prime}, \Gamma_{01}^{\prime}=\Gamma_{10}^{\prime} \exp \left(-\varepsilon_{10} / T\right)$ is the down and up interwell relaxation rate between states $|1\rangle$ and $|0\rangle$, respectively, and $T$ is the environment temperature.
In the microwave-driven cooling, states $|0\rangle$ and $|1\rangle$ are the two lowest states, which are far below other states and form a qubit. The state $|2\rangle$ is treated as an ancillary oscillator-like state, which is localized in the same well with state $|0\rangle$, and $\Delta_{12}$ is considered as the side crossover. If the qubit is in equilibrium with the environment, the population in state $|1\rangle$ is excited from state $|0\rangle$ thermally following the Boltzmann relation:

$$
p_{11} / p_{00}=\exp \left(-\varepsilon_{10} / T\right) .
$$

This relation shows that the influence of temperature is large at the detunings $\left|\varepsilon_{10}\right|<T$, which thereby becomes main cooling interests. As shown in Fig. 1(a), by repeated LZ transitions, the microwave drives the unwanted thermal population in state $|1\rangle$ to $|2\rangle$, whose population relaxes fast into state $|0\rangle$. Then the population transferred to state $|0\rangle$ faster than the thermal repopulation of state $|1\rangle$, cools the qubit. As indicated from Eq. (4), less population in state $|1\rangle$ corresponds to lower effective temperature. Hence the population in state $|1\rangle$ can determine the effective temperature realized by microwave cooling.

Microwave-driven sideband cooling depends on MDLZ transition from state $|1\rangle$ to $|2\rangle$, which exhibits a rich structure as a function of the microwave frequency. Therefore we divide the cooling into two regimes by the frequency. The first one is the coherent regime, in which the microwave frequency is larger than the decoherence rate [Fig. 2(a)]. In a driving period, the qubit state is split into the coherent superposition of states $|1\rangle$ and $|2\rangle$ by the crossover $\Delta_{12}$. Then a phase difference is constructed to form the quantum interference [18]. Especially at high frequencies near several subgigahertzes, the well resolved $n$-photon resonances are observed. For the incoherent regime the frequency is lower than the decoherence rate. The decoherence will eliminate the coherence of states $|1\rangle$ and $|2\rangle$ quickly such that the interference vanishes, as illustrated in Fig. 2(b).
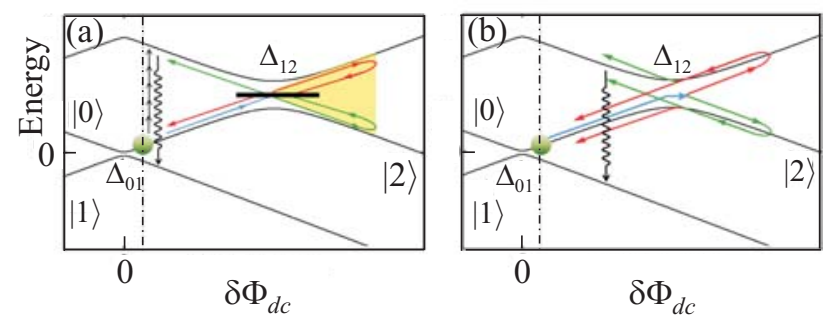

Fig. 2. (Color online) Schematic energy diagam illustrating the cooling regimes. (a) The solid line expresses the transition from state $|1\rangle$ to $|0\rangle$ at the coherent regime. The arrows mark wellresolved resonances of $n$-photon transition observed under the high-frequency driving. The shaded region corresponds to the interference phase difference. (b) The coherence interference vanishes and the transition from state $|1\rangle$ to $|0\rangle$ is realized at the incoherent transition regime. 


\section{Optimal cooling amplitude under the weak decoherence}

Now, we consider the weak decoherence where $\Gamma_{2}+\gamma \ll T$. In order to compare with the experimental results quantitatively, we choose $\Gamma_{2} / 2 \pi=0.06 \mathrm{GHz}$, $\gamma / 2 \pi=0.05 \mathrm{GHz}$ and $T=50 \mathrm{mK}$. For these parameters it was proposed that the maximal cooling near the degeneracy point can be realized at the flux amplitude $\Phi_{r f}=\Phi^{*}$ and the cooling efficiency increased monotonically with the lowering of frequency till $5 \mathrm{MHz}$ [26].

In the stationary case, $\dot{p}_{33}=\dot{p}_{22}=\dot{p}_{11}=\dot{p}_{00}=0$, we can calculate the population in state $|1\rangle$ at different detunings and frequencies using the rate equation (3). Shown in Fig. 3(a) is the population in state $|1\rangle$ as functions of detunings and frequencies for the amplitude $\Phi_{r f}=8.4 \mathrm{~m} \Phi_{0}$. For large microwave frequency [Fig. 3(b)], the population oscillates with the detuning. In order to achieve effective cooling, we have to carefully choose the frequency and detuning. This is not convenient for systems with a variety of
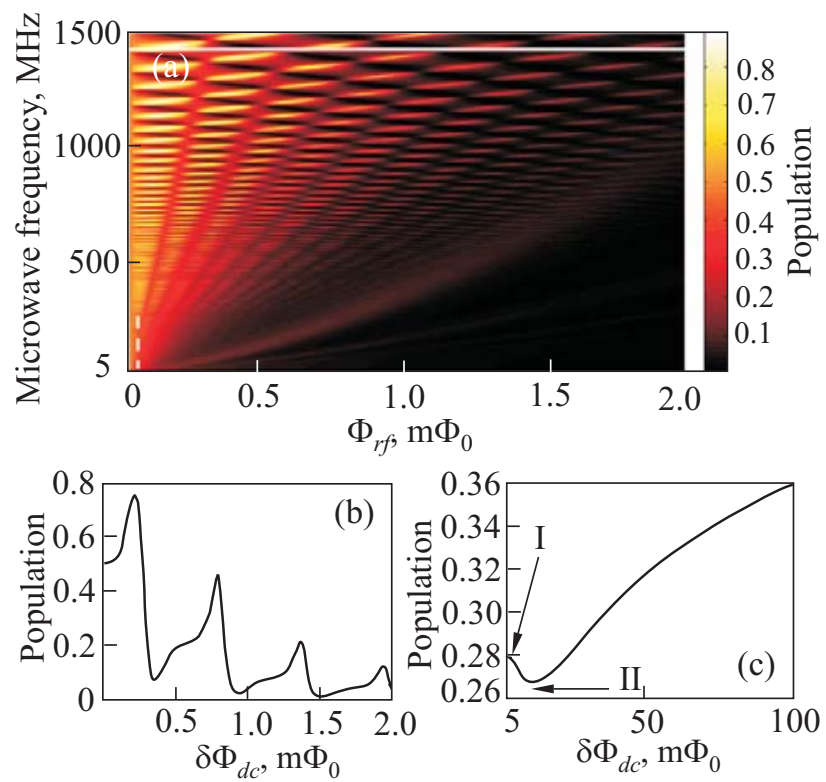

Fig. 3. (Color online) (a) The population in state $|1\rangle$ vs flux detuning and microwave frequency with the flux amplitude $\Phi_{r f}=8.4 \mathrm{~m} \Phi_{0}$. The locations of the crossovers $\Delta_{01}$ and $\Delta_{12}$ are 0 and $8.4 \mathrm{~m} \Phi_{0}$, respectively. The diabatic energy-level slope $\left|m_{0}\right|\left(\left|m_{1}\right|\right)=2 \pi \times 1.44 \mathrm{GHz} / \mathrm{m \Phi}_{0}$ and $\left|m_{2}\right|\left(\left|m_{3}\right|\right)=$ $=2 \pi \times 1.09 \mathrm{GHz} / \mathrm{m \Phi}_{0} . \quad \Delta_{01} / 2 \pi=0.013 \mathrm{GHz}, \quad \Delta_{03} / 2 \pi=$ $=\Delta_{12} / 2 \pi=0.09 \mathrm{GHz}$. The parameters above are from the experiment [42]. Furthermore we estimate $\Delta_{23} / 2 \pi=0.5 \mathrm{GHz}$. The dephasing rate we used in calculation $\Gamma_{2} / 2 \pi=0.06 \mathrm{GHz}$, the intrawell relaxation rate $\Gamma_{20} / 2 \pi=\Gamma_{31} / 2 \pi=0.1 \mathrm{GHz}$ [49], and the interwell relaxation $\Gamma_{10}^{\prime} / 2 \pi=0.00005 \mathrm{GHz}$. The environment temperature $T$ is $50 \mathrm{mK}$. (b) The population extracted from (a) in state $|1\rangle$ vs flux detuning at the frequency $1460 \mathrm{MHz}$. (c) The population extracted from (a) in state $|1\rangle$ vs the microwave frequency at the $0.05 \mathrm{~m} \Phi_{0}$. energy structures. Therefore, we pay more attention to the low-frequency region, where the incoherent transition dominates. For $\omega / 2 \pi<100 \mathrm{MHz}$ lower frequency generally leads to less population in state $|1\rangle$, which means more efficient cooling. These results agree with the experimental observations [26]. Then we extract the 1D plot, population vs microwave frequency, at a constant detuning $\delta \Phi_{d c}=0.05 \mathrm{~m} \Phi_{0}$ [marked with the white dashed line in Fig. 3(a)], shown in Fig. 3(c). Unexpectedly, as the frequency reaches $10 \mathrm{MHz}$ (point II) the population exhibits a minimum. At lower frequency $5 \mathrm{MHz}$ (point I), the population is larger than that at point II.

To refine the cooling efficiency, we calculate the population in state $|1\rangle$ as functions of microwave amplitudes and frequencies at the detuning $\delta \Phi_{d c}=0.05 \mathrm{~m} \Phi_{0}$, shown in Fig. 4(a). Then for different microwave frequencies we obtain the local minimal population, shown in Fig. 4(b). For $\omega / 2 \pi<100 \mathrm{MHz}$, the minimal population in state $|1\rangle$ decreases monotonically. The lowest population in the whole parameter space (at the detuning $\delta \Phi_{d c}=0.05 \mathrm{~m}_{0}$ ) is achieved at $5 \mathrm{MHz}$ (point III) with the corresponding amplitude $8.35 \mathrm{m \Phi}_{0}$ [Fig. 4(c)], instead of I, or II. This result suggests that the cooling efficiency in Ref. 25 could be improved by choosing the parameters given in Fig. 4 .

Then for $\omega / 2 \pi=5 \mathrm{MHz}$, we plot the population versus dc flux detuning and amplitude, shown in Fig. 5(a). At each detuning, we extract the amplitude corresponding to the minimal population, as shown in Fig. 5(b). The maximal cooling is realized with the optimal amplitude condition $\Phi_{r f} \approx \Phi^{*}-\delta \Phi_{d c}$ (quantitative result of cooling can be seen in Fig. 7). With the detuning approaching to $0 \mathrm{~m} \Phi_{0}$,
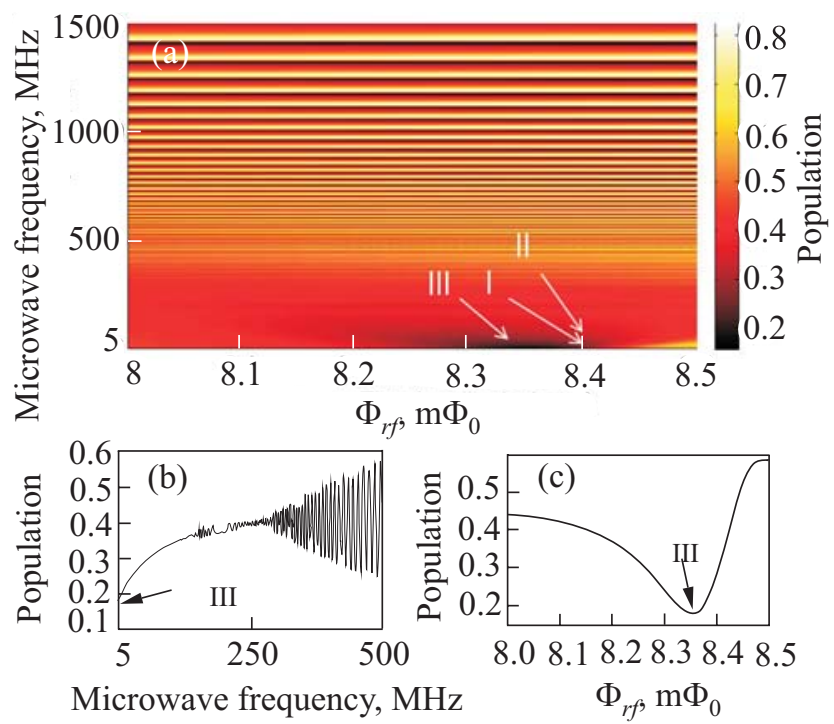

Fig. 4. (Color online) (a) The population in state $|1\rangle$ versus microwave frequency and flux amplitude with the detuning $\delta \Phi_{d c}=0.05 \mathrm{~m} \Phi_{0}$. Other parameters used are the same as those of Fig. 3. (b) The minimal population extracted from (a) at each frequency versus microwave frequency. (c) At $5 \mathrm{MHz}$, the population extracted from (a) versus flux amplitude. 

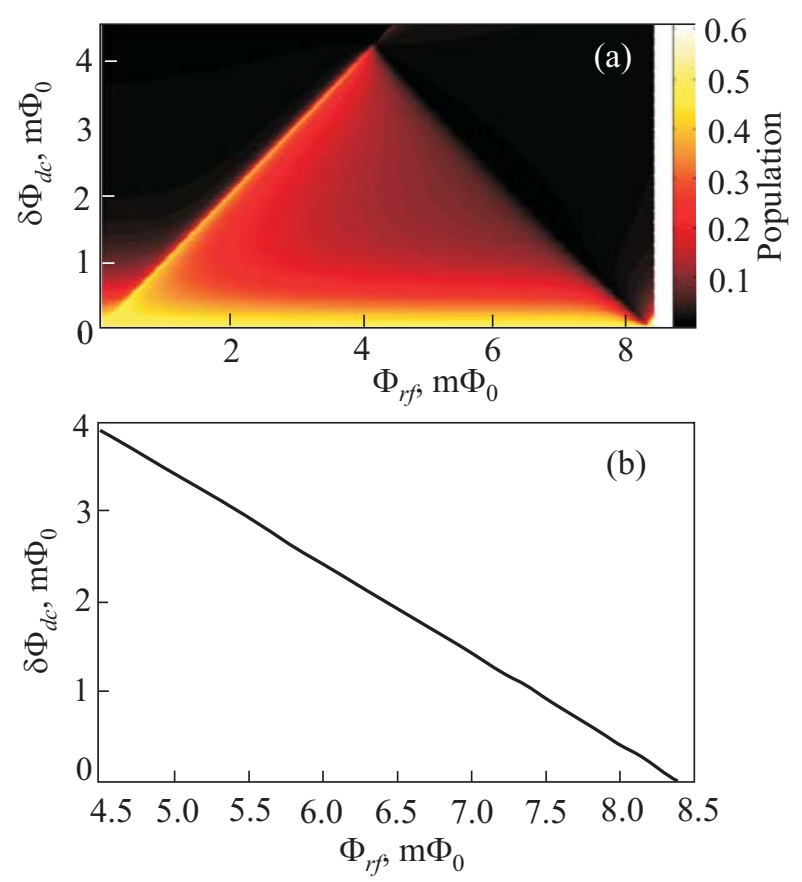

Fig. 5. (Color online) (a) The population in state $|1\rangle$ versus flux detuning and microwave flux amplitude with the frequency $5 \mathrm{MHz}$. Other parameters are identical with those of Fig. 3. (b) The amplitude corresponding to the minimal population extracted from (a) at each detuning.

the amplitude condition can be changed to $\Phi_{r f}=\Phi^{*}$, just as those demonstrated in Ref. 25.

We can generalize the amplitude condition for optimal cooling at weak decoherence. In the stationary case, considering $W_{12}, W_{03} \ll \Gamma_{31}, \Gamma_{20}$, the population in state $|1\rangle$ is given by

$$
p_{11}=\frac{\Gamma_{01}^{\prime}+W_{03}}{\Gamma_{10}^{\prime}+\Gamma_{01}^{\prime}+W_{12}+W_{03}} .
$$

When the dc flux detuning is large, $W_{12}, \Gamma_{01}^{\prime} \gg W_{03}$. Therefore $W_{03}$ can be neglected in Eq. (5) and the population in state $|1\rangle$ would depend on $W_{12}$. Since $W_{12}$ represents the population transferred from state $|1\rangle$ to $|2\rangle$ in unit time, it relies not only on the population transferred through LZ transition in the crossover $\Delta_{12}$ every time, but also the microwave frequency which determines the frequency of LZ transitions. When the system stays at the particular dc flux detuning, the tunneling transition between states $|1\rangle$ and $|2\rangle$ is given by $[55,56]$

$$
\Gamma=\frac{\Delta_{12}^{2}}{2} \frac{\left(\Gamma_{2}+\gamma\right)}{\varepsilon_{12}^{2}+\left(\Gamma_{2}+\gamma\right)^{2}},
$$

where $\gamma=\Gamma_{20} / 2$. Equation (6) indicates that there is nearly no tunneling transition between states $|1\rangle$ and $|2\rangle$ for the detuning far away from $\Delta_{12}$. The tunneling can be considered in a transition region $\left|\varepsilon_{12}\right| \lesssim \Gamma_{2}+\gamma$, where $\Gamma \sim \Delta_{12}^{2} /\left[2\left(\Gamma_{2}+\gamma\right)\right]$. Therefore the longer time staying in the transition region in one period, the larger population transferred from state $|1\rangle$ to $|2\rangle$, and hence the better cooling efficiency. However, the long time staying in the transition region requires a slow sweeping velocity. Since the velocity of a sinusoidal movement approaches to zero at its full amplitude, the amplitude which can exactly bring the system to the transition region will result the largest transition rate from state $|1\rangle$ to $|2\rangle$. When the decoherence is weak, the transition region is always near the crossover. As a result, we have the optimal amplitude condition $\Phi_{r f} \approx \Phi^{*}-\delta \Phi_{d c}$ as obtained above.

\section{Improved cooling method under the strong decoherence}

Then, we discuss the boundary for the flux detuning to realize effective microwave cooling. As shown in Eq. (4), the upper limit for this boundary is defined by the environment temperature $T$. The interests needed to be cooled down span in the detunings $\left|\varepsilon_{01}\right| \lesssim T$, above which the population on the excited state is already very small. The lower limit is set by the decoherence. For weak decoherence, LZ transition happens almost right at each crossover [57]. If we initially bias the system at $\delta \Phi_{d c}$, the optimal amplitude condition can be achieved when $\Phi_{r f} \approx \Phi^{*}-\delta \Phi_{d c}$. Further increasing the microwave amplitude will degrade the cooling efficiency. Due to the symmetric energy diagram, higher state $|3\rangle$, acting as another oscillator-like state, will involve into the dynamics, transferring the population from state $|0\rangle$ to $|3\rangle$ when the amplitude reaches the crossover $\Delta_{03}$, i.e., $\Phi_{r f}>\delta \Phi_{d c}+\Phi^{*}$. With the driving on, the system will relax to $|1\rangle$ from $|3\rangle$ continuously and the cooling effect is very weak. Fortunately one can control microwave amplitude to avoid reaching the crossover $\Delta_{03}$ in the cooling cycle since the optimal amplitude $\Phi_{r f}$ is always smaller than $\delta \Phi_{d c}+\Phi^{*}$. However, for strong decoherence we have different situation. According to Eq. (6), the decoherence widens the transition region to $\Gamma_{2}+\gamma$. Now when $\Gamma_{2}+\gamma=\left(\left|m_{0}\right|+\left|m_{3}\right|\right)\left(\delta \Phi_{d c}+\Phi^{*}-\Phi_{r f}\right)$, LZ transition to $|3\rangle$ will occur. When $\Gamma_{2}+\gamma>2 \delta \Phi_{d c}\left(\left|m_{0}\right|+\left|m_{3}\right|\right)$, the optimal cooling cannot be fulfilled anymore. Therefore, lower limit of the dc detuning is $\left(\Gamma_{2}+\gamma\right) / 2$ (we have taken approximation $m_{3} \approx m_{1}$ ).

With the decoherence rate increasing, the lower limit moves toward the upper limit $T$. Hence, we define weak decoherence as $\Gamma_{2}+\gamma \ll T$ ( $\Delta_{03}$ will have little influence in the ordinary cooling method) and strong decoherence as $\Gamma_{2}+\gamma \sim T$ ( $\Delta_{03}$ will destroy the cooling). 
Shown in Fig. 6(a) is the contour plot of the population in state $|1\rangle$ at the detuning $\delta \Phi_{d c}=0.05 \mathrm{~m} \Phi_{0}$ with different amplitudes and frequencies for strong decoherence. Following the same procedure used in Fig. 4, we extract the minimal population for each frequency [Fig. 6(b)]. It is found that the decoherence makes the population near 0.5 , indicating a very inefficient cooling. In Fig. 6(c) we show that at the detuning $0.05 \mathrm{~m} \Phi_{0}$ and $5 \mathrm{MHz}$, the lowest population is obtained at $8.1 \mathrm{~m} \Phi_{0}$, different from the amplitude condition we obtained in Sec. 3. Strong decoherence will extend transition region near $\Delta_{12}$ and larger amplitude is needed to realize better cooling. However the transition region of $\Delta_{03}$ is also widened. Larger amplitude will drive the state closer to $\Delta_{03}$, generating the population transferred from state $|0\rangle$ to $|1\rangle$ with the relaxation thus damaging the cooling efficiency. Therefore, a slightly lower amplitude comparing with the optimal amplitude in weak decoherence will minimize the effect of state $|3\rangle$.

Then changing the detuning and repeating the procedure that generates Fig. 6(b), we obtain the minimal population in state $|1\rangle$ as functions of detunings and frequencies, shown in Fig. 7(a). The cooling efficiency increased monotonically with the lowering of frequency till $5 \mathrm{MHz}$. From Fig. 7(a) we extract the 1D plot of the population vs detuning at $5 \mathrm{MHz}$, shown as the dotted line in Fig. 7(b). For strong decoherence, the population after microwave cooling is close to or even worse than the population in the equilibrium, indicating almost no cooling effect. We also plot the minimal population at $5 \mathrm{MHz}$ under the weak decoherence, i.e., $\Gamma_{2} / 2 \pi=0.06$ $\mathrm{GHz}$, shown as the dashed line. The effective temperature
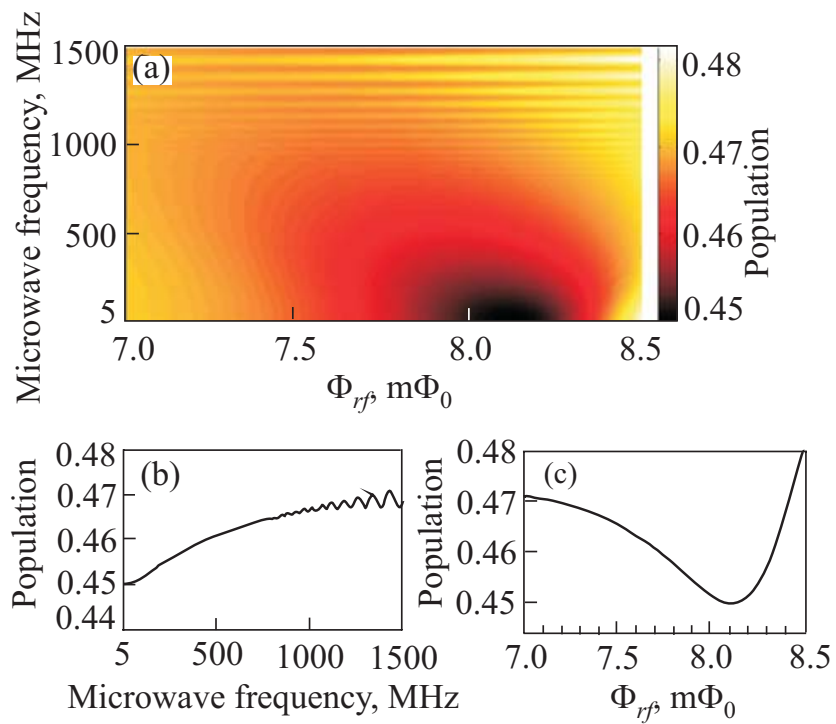

Fig. 6. (Color online) (a) The population in state $|1\rangle$ versus microwave frequency and flux amplitude with the detuning $\delta \Phi_{d c}=0.05 \mathrm{~m}_{0}$ and the dephasing rate $\Gamma_{2} / 2 \pi=1 \mathrm{GHz}$. Other parameters used are the same as those of Fig. 3. (b) For each frequency from (a), we find the minimal population on microwave flux amplitude axis. (c) We keep frequency fixed at $5 \mathrm{MHz}$, plot the population as a function of flux amplitude.
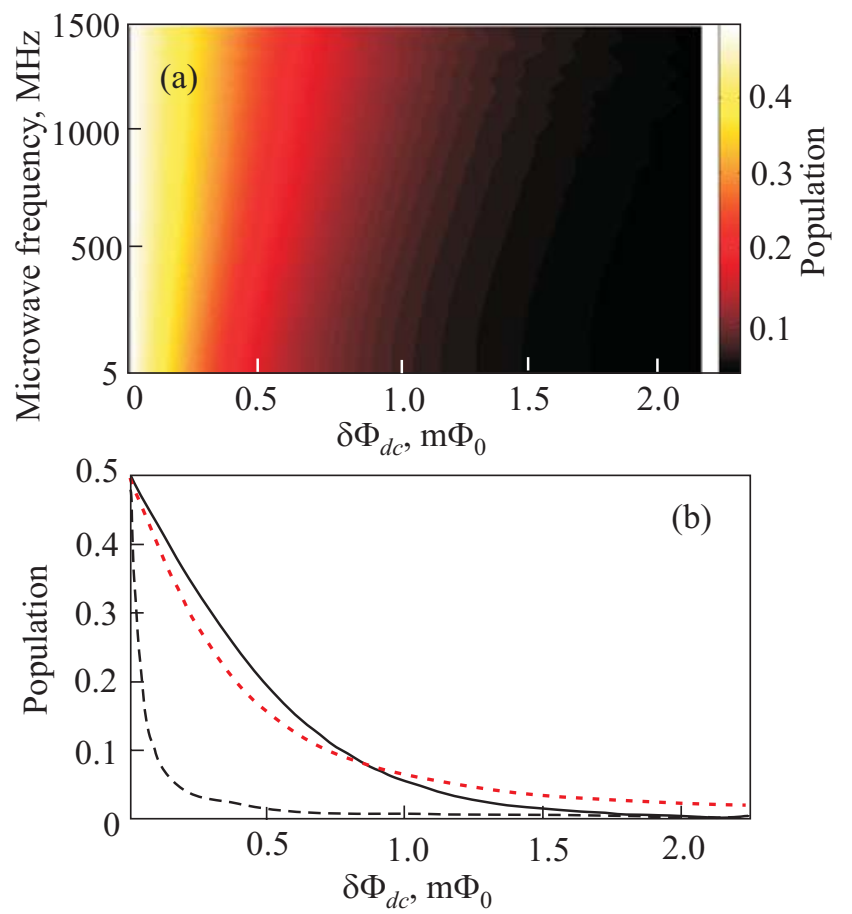

Fig. 7. (Color online) (a) The minimal population in state $|1\rangle$ among different flux amplitudes versus the frequency and detuning under the dephasing rate $\Gamma_{2} / 2 \pi=1 \mathrm{GHz}$. Other parameters used are the same as those of Fig. 3. (b) The comparison between the minimal population in state $|1\rangle$ at $5 \mathrm{MHz}$ for each detuning under different dephasing rates (dashed line corresponds to $\Gamma_{2} / 2 \pi=0.06 \mathrm{GHz}$ while dotted line corresponds to $\Gamma_{2} / 2 \pi=1$ $\mathrm{GHz}$ ). The solid line represents the population in the equilibrium with the temperature $50 \mathrm{mK}$.

obtained from the best fit is about $5 \mathrm{mK}$. Figure 7(b) shows that for strong decoherence another oscillator-like state $|3\rangle$ involved greatly cancels the effect of original oscillator-like state $|2\rangle$. Therefore, better technique is needed for the efficient cooling.

We found that the cooling efficiency can be dramatically increased by changing microwave wave form. In the first half period of the ordinary cooling cycle, the sinusoidal microwave drives the state in the direction to the side crossover $\Delta_{12}$, and after going back to the initial detuning, the state is driven in the direction to another side crossover $\Delta_{03}$. Only the latter half period brings the coupling to state $|3\rangle$ thus degrading cooling effect. Therefore if we cancel the second half period of the waveform, the cooling efficiency will increase. This means that when the state goes back to the initial position, the microwave drives it in the direction to $\Delta_{12}$ instead of $\Delta_{03}$. This minor change remarkably increases the efficiency to the cooling. It is worth to point out that the technique is readily available [45], making our method practical. Of course, one can also use periodic triangular waveform to drive the system. But for the convenience of calculation, we still use the sinusoidal waveform shown in Fig. 1(a). Now MDLZ transition rate can be changed to 


$$
W_{j i}=W_{i j}=\frac{\Delta_{i j}^{2}}{2} \sum_{n} \frac{\left(\Gamma_{2}+\gamma\right) J_{n}^{2}\left(A_{i j} / \omega\right)}{\left(\varepsilon_{i j}+A_{i j}-n \omega\right)^{2}+\left(\Gamma_{2}+\gamma\right)^{2}},
$$

where state $|i\rangle$ and $|j\rangle$ are in the left and right well, respectively, and $\varepsilon_{i j}$ has the same definition as Eq. (2). Furthermore, $\Gamma_{01}^{\prime}=\Gamma_{10}^{\prime} \exp \left\{-\left(\varepsilon_{10}+A_{10}\right) / T\right\}$.

We compare the cooling effect of two methods in Fig. 8. With the frequency $5 \mathrm{MHz}$ and $\Gamma_{2} / 2 \pi=1 \mathrm{GHz}$, we employ the ordinary cooling method to obtain the population in state $|1\rangle$ at different amplitudes and detunings, as shown in Fig. 8(a). Figure 8(b) is the contour plot of the population in state $|1\rangle$ with the improved method at the same condition as that in Fig. 8(a). For each detuning, we extract the minimal population in Fig. 8(b) and its corresponding amplitude, shown in Figs. 8(c) and (d), respectively.
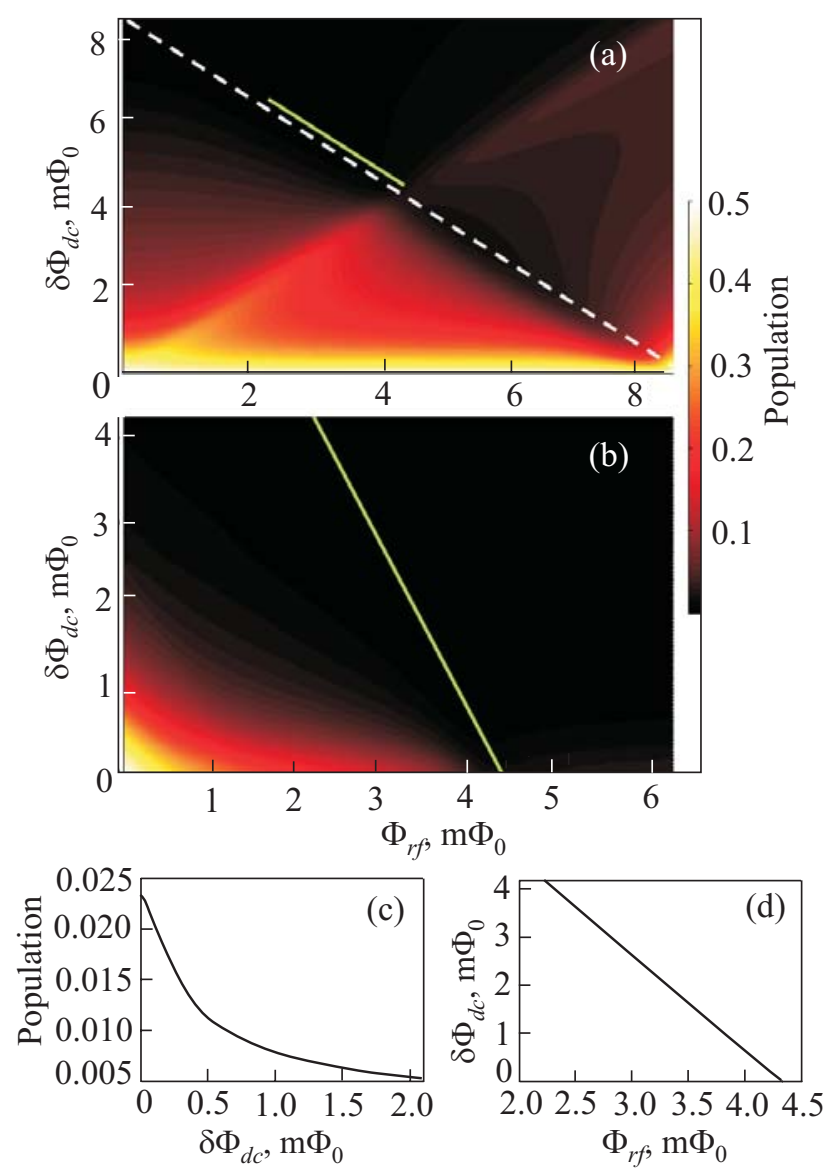

Fig. 8. (Color online) (a) The population in state $|1\rangle$ versus flux amplitude and detuning with the frequency $5 \mathrm{MHz}$ and the dephasing rate $\Gamma_{2} / 2 \pi=1 \mathrm{GHz}$ in the original cooling method. Other parameters are identical with those of Fig. 3 . The white dashed line indicates the optimal cooling region in the original method. (b) The population in state $|1\rangle$ versus the flux amplitude and detuning under the same condition with (a) in the improved method. The green solid line expresses the optimal cooling region in the improved method. (c) and (d) The minimal population and the corresponding amplitude extracted from (b) at each flux detuning.
One can see that the cooling efficiency in the improved method is much better than that in the ordinary method shown in Fig. 7(b), especially at the detuning near $0 \mathrm{~m} \Phi_{0}$. Moreover, without the influence of $\Delta_{03}$, the change of waveform makes the optimal amplitude now needs to satisfy the condition $2 \Phi_{r f}+\delta \Phi_{d c} \approx \Phi^{*}$.

In order to compare the optimal amplitude conditions of two methods, we mark them in Fig. 8(a) and (b). The white dashed line in Fig. 8(a) represents the optimal amplitude in the ordinary method, while the green solid line in Fig. 8(b) represents that in the improved method. We also redraw the green solid line in Fig. 8(a), keeping the same amplitude scale and cooling efficiency (or population). Actually, the green line almost overlaps the optimal cooling region in the ordinary method. However, for the same amplitude, the detunings (which represents cooling interests) are different. In the ordinary method, the optimal cooling region with particular amplitude is realized at large detuning (about 4-6 $m \Phi_{0}$ ) [Fig. 8(a)] which is not sensitive to the disturbance of the strong thermal noise. The population is already very low and the cooling is not necessary. However, in the improved method, the region transforms to low detuning (about $0-4 m \Phi_{0}$ ) which is bothered by the temperature, with almost doubled effective cooling region [Fig. 8(b)]. Under the strong decoherence, the advantage of the improved method is remarkable. It enables us to cool efficiently the interests at low detuning which need be cooled down mostly, through redistributing of cooling resource.

This robustness of the cooling method can effectively cool down the qubit and reduce the decoherence which initially might be strong. Usually, the strong decoherence accompanies the large geometrical size of the qubit $[43,58]$. Based on the improved method, large size devices, such as rf superconducting quantum interference device, plays the role of cooling center and provides the potential application in refrigerating more environmental degrees of freedom [59] and more neighboring quantum systems [60].

\section{Optimal cooling frequency}

Following the procedure used in Fig. 4, at the detuning $\delta \Phi_{d c}=0.05 \mathrm{~m} \Phi_{0}$, we change the amplitude and frequency to obtain the population in state $|1\rangle$ for the improved method [Fig. 9(a)]. Then we extract the minimal population at each frequency in Fig. 9(a), shown in Fig. 9(b). Contrary to the behavior in Fig. 4(b), we find larger frequency yields better cooling. To explain this interesting phenomenon, we will mainly investigate the frequency for optimal cooling. At first we discuss the improved method.

As indicated in Eq. (5), the transition rate $W_{12}$ is the main factor influencing the cooling. The maximal transition rate $W_{12}$ contributes to the minimal population in state $|1\rangle$. Hence we start from MDLZ transition under the 


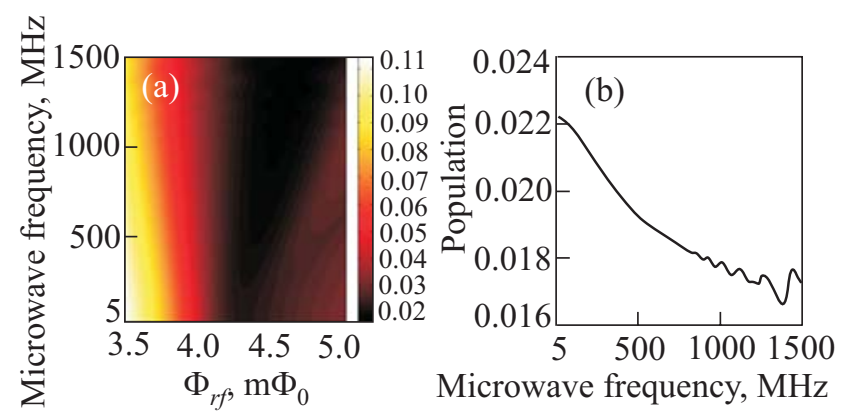

Fig. 9. (Color online) (a) The population in state $|1\rangle$ versus the frequency and flux amplitude with the detuning $\delta \Phi_{d c}=$ $=0.05 \mathrm{m \Phi}_{0}$ and the dephasing rate $\Gamma_{2} / 2 \pi=1 \mathrm{GHz}$ in the improved method. Other parameters used are the same as those of Fig. 3. (b) The minimal population in state $|1\rangle$ extracted from (a) at each microwave frequency.

improved method and change the transition rate Eq. (7) to the form

$$
W_{i j}=\frac{\Delta_{i j}^{2}}{2} \sum_{n} \frac{\left(2 \omega / A_{i j}\right)^{2 / 3} \mathrm{Ai}^{2}\left[\left(2 \omega / A_{i j}\right)^{1 / 3}\left(n-A_{i j} / \omega\right)\right]}{\left[\varepsilon_{i j}+A_{i j}+n \omega\right]^{2} /\left(\Gamma_{2}+\gamma\right)+\Gamma_{2}+\gamma},
$$

where we use the Airy function $\operatorname{Ai}(x)$ to express the Bessel function $J_{n}(x)=(2 / x)^{1 / 3} \operatorname{Ai}\left[(2 / x)^{1 / 3}(n-x)\right]$. The sum is determined by the terms with $n$, the nearest integer to $\left(\varepsilon_{i j}+A_{i j}\right) / \omega$. Therefore, we have

$$
\begin{aligned}
& W_{i j} \approx \Delta_{i j}^{2} \frac{\omega^{2 / 3} A^{-2 / 3}}{2} \mathrm{Ai}^{2}\left[\left(\frac{2 \omega}{A}\right)^{1 / 3} \frac{-\varepsilon_{i j}-2 A_{i j}}{\omega}\right] /\left(\Gamma_{2}+\gamma\right)+ \\
& +\Delta_{i j}^{2} \frac{A^{-2 / 3}}{2} \mathrm{Ai}^{2}\left[\left(\frac{2 \omega}{A}\right)^{1 / 3} \frac{-\varepsilon_{i j}-2 A_{i j}-\omega}{\omega}\right] /\left(\frac{\omega^{4 / 3}}{\Gamma_{2}+\gamma}+\frac{\Gamma_{2}+\gamma}{\omega^{2 / 3}}\right)+ \\
& +\Delta_{i j}^{2} \frac{A^{-2 / 3}}{2} \operatorname{Ai}^{2}\left[\left(\frac{2 \omega}{A}\right)^{1 / 3} \frac{-\varepsilon_{i j}-2 A_{i j}+\omega}{\omega}\right] /\left(\frac{\omega^{4 / 3}}{\Gamma_{2}+\gamma}+\frac{\Gamma_{2}+\gamma}{\omega^{2 / 3}}\right) .
\end{aligned}
$$

From the above expression we can qualitative understand the physics picture. Resulting from the contribution of the factor $\left[\omega^{4 / 3} /\left(\Gamma_{2}+\gamma\right)\right]+\left[\left(\Gamma_{2}+\gamma\right) / \omega^{2 / 3}\right]$, the second and third term of Eq. (9) will increase with microwave frequency until the frequency reaches about $\left(\Gamma_{2}+\gamma\right) / 2 \pi$. In this process the total transition rate will also increase. This explains why under the strong decoherence the increase of frequency from 5 to $500 \mathrm{MHz}$ reduces the population in Fig. 9(b). When the frequency is further increased above $\left(\Gamma_{2}+\gamma\right) / 2 \pi$, this term will lead to the decrease of $W_{i j}$. Therefore the frequency corresponding to the maximum of $W_{i j}$ relies on the decoherence rate. Since the contribution from other terms in Eq. (8) is neglected here, it is only a qualitative analysis and further conclusions could not be obtained from Eq. (9). In addition, as shown in Eq. (9), the maximum of $W_{i j}$ also requires $-\varepsilon_{i j}-2 A_{i j} \approx 0$, which is exactly the result of optimal amplitude relation in the improved method in Sec. 4.

For quantitative description, we need to return to the expression in Eq. (7). For the weak decoherence, e.g., $\Gamma_{2}+\gamma=2 \pi \times 0.1 \mathrm{GHz}$, the frequency corresponding to the maximum of $W_{i j}$ will be nearly $10 \mathrm{MHz}$ [Fig. 10(a)]. If the decoherence is strong, e.g., $\Gamma_{2}+\gamma=2 \pi \times 1.05 \mathrm{GHz}$, the maximum of $W_{i j}$ will be obtained at a large frequency nearly $470 \mathrm{MHz}$, as shown in Fig. 10(b). Figure 10(c) shows the decoherence rate vs the frequency corresponding to the maximum of $W_{i j}$. These frequencies are at the incoherent regime defined in Sec. 2. And they increase linearly with the decoherence rate, agreeing with the prediction of
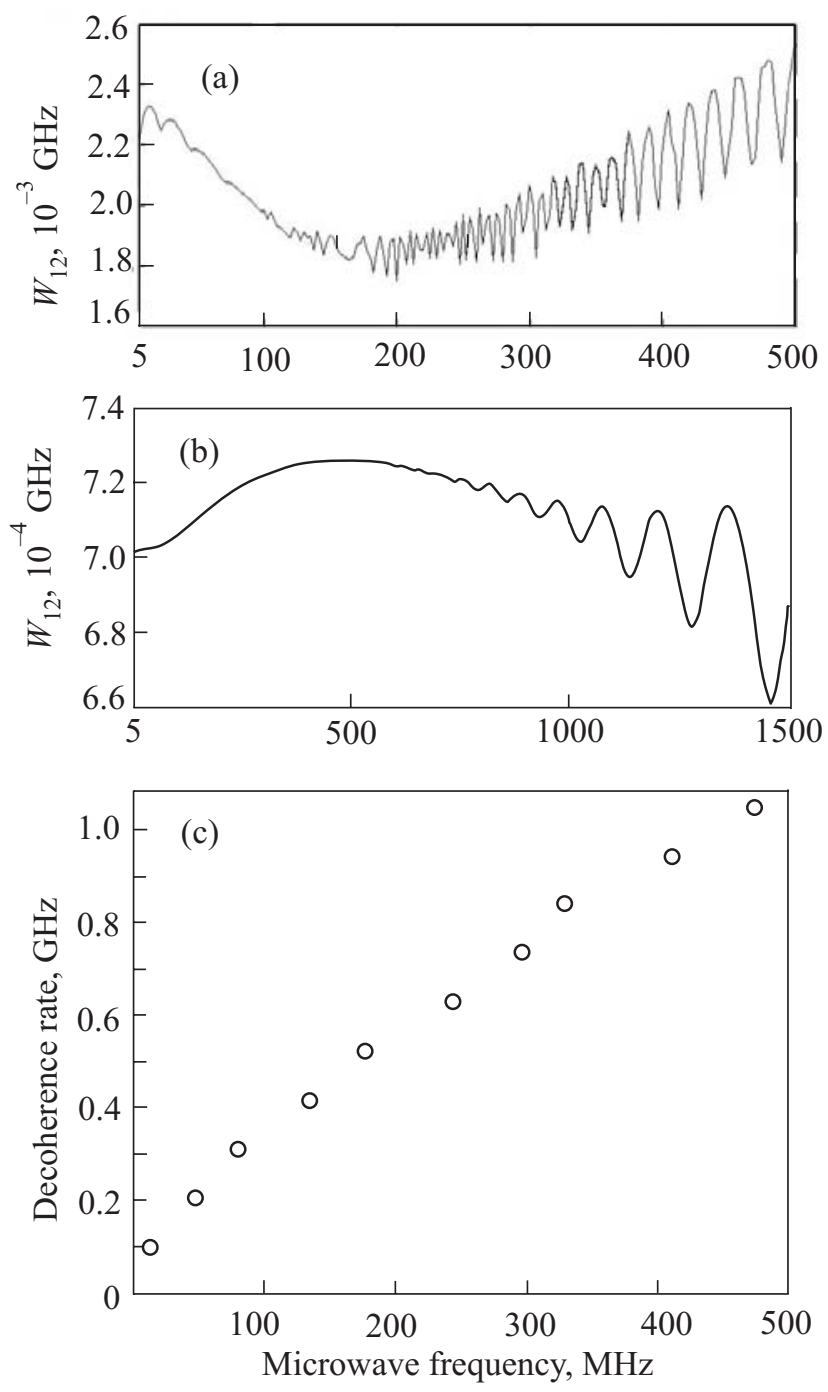

Fig. 10. (a) and (b) The maximum of $W_{12}$ among different amplitudes at each frequency at the detuning $\delta \Phi_{d c}=0.05 \mathrm{~m} \Phi_{0}$ under the dephasing rate $\Gamma_{2} / 2 \pi=0.05$ and 1 , respectively. (c) The decoherence rate versus the frequency of the maximum of $W_{12}$ at the detuning $\delta \Phi_{d c}=0.05 \mathrm{~m} \Phi_{0}$. All figures are calculated in the improved method. Other parameters are the same as those of Fig. 3. 
qualitative analysis. In addition, as illustrated in the discussion of Eq. (9), the optimal frequency tuned by decoherence is almost independent of the dc detuning. To clarify this point, we change the dc detuning for the unchanged decoherence. Our calculation shows that the frequency corresponding to the maximum of $W_{i j}$ is nearly constant at different detunings. It means one can use a constant microwave frequency to realize the maximal MDLZ transition rate.

MDLZ transition rate $W_{i j}$ depends on the driving frequency as well as the population transferred through LZ transition during one period. Both factors are related with the microwave frequency. Larger frequency generates more cycles of LZ transitions in unit time. On the other hand, it also results into larger velocity thus shorter time in the transition region, leading to less LZ transition popula-
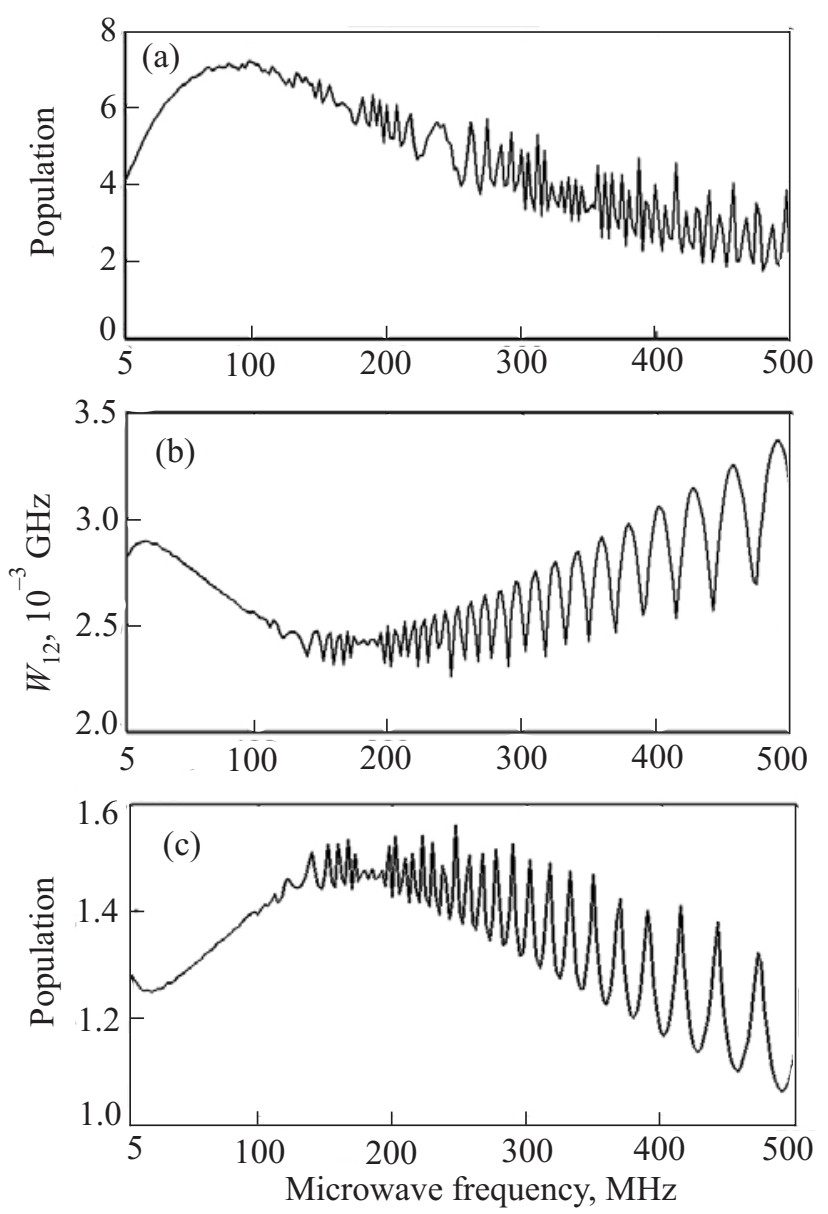

Fig. 11. (a) The minimal population in state $|1\rangle$ among different amplitudes at each frequency with the detuning $\delta \Phi_{d c}=0.05 \mathrm{~m} \Phi_{0}$ and the dephasing rate $\Gamma_{2} / 2 \pi=0.05 \mathrm{GHz}$. (b) The maximum of $W_{12}$ among different amplitudes at each frequency with the detuning $\delta \Phi_{d c}=0.05 \mathrm{~m} \Phi_{0}$ and the dephasing rate $\Gamma_{2} / 2 \pi=0.05 \mathrm{GHz}$. (c) The minimal population in state $|1\rangle$ among different amplitudes at each frequency with the detuning $\delta \Phi_{d c}=3 \mathrm{~m} \Phi_{0}$ and the dephasing rate $\Gamma_{2} / 2 \pi=0.05 \mathrm{GHz}$. All figures are calculated in the improved method. Other parameters are the same as those of Fig. 3. tion as given in Eqs. (1) and (6). The above qualitative and quantitative discussions indicate that at an appropriate frequency the maximum of MDLZ transition rate can be obtained. Moreover, the decoherence has an important contribution to the tuning of this frequency.

For the improved method, besides maximal MDLZ transition rate, two additional factors may affect the population of the system. One of them is the effect of other crossovers. In Fig. 11(a), we plot the minimal population in state $|1\rangle$ versus the frequency for $\Gamma_{2} / 2 \pi=0.05 \mathrm{GHz}$ and $\delta \Phi_{d c}=0.05 \mathrm{~m} \Phi_{0}$. At low frequency, the population does not decrease to a minimum at about $10 \mathrm{MHz}$ as that $W_{12}$ displays in Fig. 10(a). A monotonic increase to $100 \mathrm{MHz}$ is observed instead. For small detuning, the low driving frequency will results in the low velocity near $\Delta_{01}$ and $\Delta_{23}$. Therefore both $W_{01}$ and $W_{23}$ have contribution on the population in state $|1\rangle$. If we choose the detuning far away from the degeneracy point, this difference between the population in state $|1\rangle$ and $W_{12}$ would disappear as shown in Figs. 11(b) and (c).

Another factor that will affect the population is the interwell relaxation. We find that when the decoherence rate is large, e.g., $\Gamma_{2} / 2 \pi=1 \mathrm{GHz}$, the population exhibits a monotonic decrease [Fig. 9(b)] with the frequency. However, $W_{12}$ shows an increase at the low frequency and decrease at high frequency [Fig. 10(b)]. This is because of the interwell relaxation. At the detuning far away from $0 \mathrm{~m} \Phi_{0}$, the population in state $|1\rangle$ is

$$
p_{11}=\frac{\Gamma_{01}^{\prime}}{\Gamma_{10}^{\prime}+\Gamma_{01}^{\prime}+W_{12}},
$$

where $\Gamma_{01}^{\prime}=\Gamma_{10}^{\prime} \exp \left\{-\left(\varepsilon_{10}+A_{10}\right) / T\right\}$, and $W_{i j}$ is defined by Eq. (7). In order to obtain the optimal cooling, larger

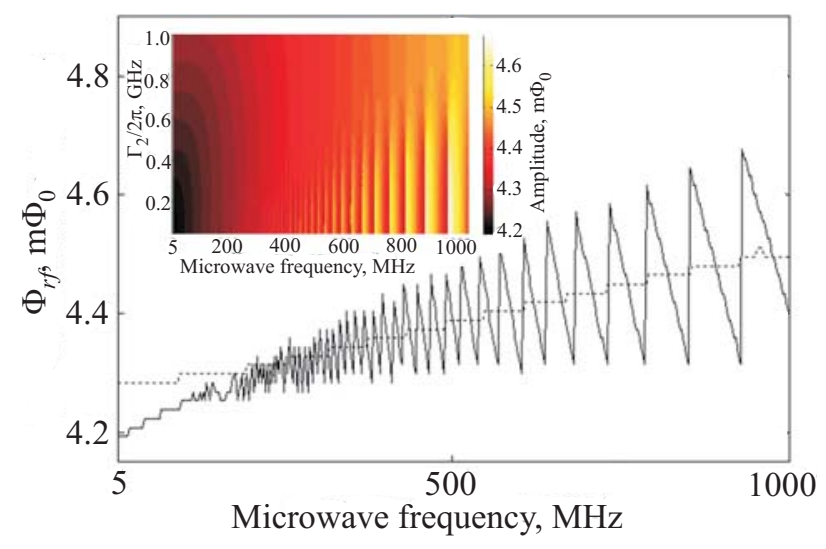

Fig. 12. (Color online) The amplitude corresponding to the maximum of $W_{12}$ at each frequency in the improved method with the detuning $\delta \Phi_{d c}=0.05 \mathrm{~m}_{0}$ at $\Gamma_{2} / 2 \pi=0.05 \mathrm{GHz}$ (the solid line) and $1 \mathrm{GHz}$ (the dotted line). Other parameters used here are identical with those of Fig. 3. The inset shows the amplitude corresponding to the maximum of $W_{12}$ at each dephasing rate and frequency with the detuning $\delta \Phi_{d c}=0.05 \mathrm{~m} \Phi_{0}$. 
frequency requires larger amplitude (Fig. 12) [61]. However, according to the expression of $\Gamma_{01}^{\prime}$ in Eq. (10), larger amplitude will lead to a smaller $\Gamma_{01}^{\prime}$, and the population in state $|1\rangle$ decreases with $\Gamma_{01}^{\prime}$. Therefore, the final population exhibits different characters from $W_{12}$.

For the ordinary cooling method, $\Delta_{03}$, instead of interwell relaxation and the crossovers at the degeneracy point $\left(\Delta_{01}\right.$ and $\left.\Delta_{23}\right)$ will affect cooling. However, the decoherence induced maximal MDLZ transition still exists. Quantitatively, now the Eq. (8) is invalid and we have to use Eq. (2). With $\varepsilon_{i j}+A_{i j}$ replaced by $\varepsilon_{i j}$, following the same procedure we can obtain a similar result of Eq. (9), in the amplitude and frequency conditions for maximal MDLZ transition. Under the weak decoherence, if we choose the large detuning far away from $\Delta_{01}$ [62], only $W_{12}$ dominates cooling and the maximum of $W_{12}$ results the optimal cooling. As shown in Fig. 13(a), at $\delta \Phi_{d c}=0.5 \mathrm{~m} \Phi_{0}$, we change microwave amplitude at each frequency to obtain the minimal population. A nonmonotonic behavior of population vs microwave frequency can be found, with the maximal cooling achieved at the frequency about $10 \mathrm{MHz}$. However, if the detuning is too small, as shown in Fig. 4(b), we cannot observe the minimum due to the presence of $\Delta_{03}$. When we only consider $W_{12}$, a maximum of transition rate [Fig. 13(b)] will appear again.
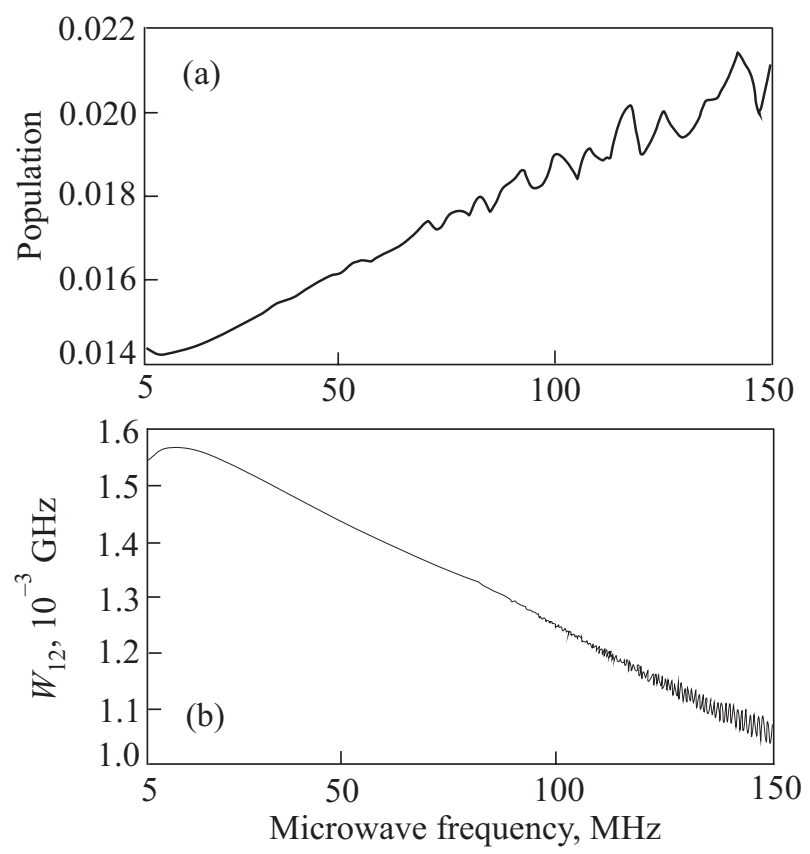

Fig. 13. (a) The minimal population in state $|1\rangle$ among different amplitudes at $5 \mathrm{MHz}$ with the detuning $\delta \Phi_{d c}=0.05 \mathrm{~m} \Phi_{0}$ and dephasing rate $\Gamma_{2} / 2 \pi=0.05 \mathrm{GHz}$ in the ordinary method. (b) The maximum of $W_{12}$ among different amplitudes at each frequency with the detuning $\delta \Phi_{d c}=0.05 \mathrm{~m} \Phi_{0}$ and the dephasing rate $\Gamma_{2} / 2 \pi=0.06 \mathrm{GHz}$ in the ordinary method. Other parameters used are the same as those of Fig. 3.
In addition, we would like to emphasize that microwave cooling is ultimately limited by the environment temperature and microwave frequency. At sufficient high temperature and low frequency, i.e., $\Gamma_{10}^{\prime} \exp \left(-\varepsilon_{10} / T\right)>\omega$, it is obviously that the population transfer due to the microwave cooling pulse, which competes with the thermal transfer, will not be fast enough to compensate the latter contribution, resulting in a less efficient cooling at low frequencies.

\section{Conclusion}

Starting from a recent experiment, we investigate the microwave-driven cooling in an artificial atom subjected to the environment noise. Under the weak decoherence, we show the optimal cooling requires that the amplitude satisfies the relation $\Phi_{r f} \approx \Phi^{*}-\delta \Phi_{d c}$.

We further discussed the cooling under the strong decoherence, when more vibrational degrees of freedom are coupled to the two lowest states, making the ordinary cooling method ineffective. To recover the effective cooling, we proposed an improved cooling method, which employs a revised driving waveform to avoid "harming" side crossover. This method can be used to improve the cooling efficiency, prepare the quantum state and suppress decoherence in multi-qubit system under not only the weak decoherence but also the strong decoherence. Especially, for systems consisting of the interaction between the qubit and the real resonator, such as micromechanical beam (resonator), this method can also be used to cool down the qubit, or even resonators. In addition, only small microwave frequency is needed in the improved method, thus providing the possibility to use our method on adiabatic quantum computation.

In addition, we investigated the optimal frequency for efficient cooling. We demonstrated that lower frequency will not result in better cooling effect and the frequency condition for optimal cooling is complicated. An essential element that determines the optimal cooling frequency is MDLZ transition rate. Our study demonstrates qualitatively and quantitatively the frequency where MDLZ transition rate reaches maximum is tuned by the decoherence and monotonically increases with the decoherence rate, deepening the understanding to the physical mechanism of MDLZ transition as well as the optimal cooling. Currently, MDLZ transition in the coherent regime has attracted lots of attentions due to coherent phenomena. We point out the incoherent regime has the particular advantage in quantum cooling. Furthermore, the results based on MDLZ transition can be applied to other systems with similar energy states structure.

\section{Acknowledgments}

We thank S.O. Valenzuela for useful discussions. This work was partly supported by the State Key Pro- 
gram for Basic Researches of China (2011CB922104, 2011CBA00205), the NSFC (91021003), the Natural Science Foundation of Jiangsu Province (BK2010012), and PAPD.

1. Y. Makhlin, G. Schön, and A. Shnirman, Rev. Mod. Phys. 73, 357 (2001).

2. M.A. Nielsen and I.L. Chuang, Quantum Computation and Quantum Information, Cambridge University Press, Cambridge (2000).

3. J.Q. You and F. Nori, Phys. Today 58, No. 11, 42 (2005).

4. H. Mooij, Science 307, 1210 (2005).

5. J. Clarke and F.K. Wilhelm, Nature (London) 453, 1031 (2008).

6. G. Wendin and V.S. Shumeiko, Fiz. Nizk. Temp. 33, 957 (2007) [Low Temp. Phys. 33, 724 (2007)].

7. Y. Nakamura, Y.A. Pashkin, and J.S. Tsai, Nature (London) 398, 786 (1999).

8. D. Vion, A. Aassime, A. Cottet, P. Joyez, H. Pothier, C. Urbina, D. Esteve, and M.H. Devoret, Science 296, 886 (2002).

9. Y. Yu, S.Y. Han, X. Chu, S.I. Chu, and Z. Wang, Science 296, 889 (2002).

10. J.M. Martinis, S. Nam, J. Aumentado, and C. Urbina, Phys. Rev. Lett. 89, 117901 (2002).

11. I. Chiorescu, Y. Nakamura, C.J.P.M. Harmans, and J.E. Mooij, Science 299, 1869 (2003).

12. Y. Nakamura, Y.A. Pashkin, and J.S. Tsai, Phys. Rev. Lett. 87, 246601 (2001).

13. I. Chiorescu, P. Bertet, K. Semba, Y. Nakamura, C.J.P.M. Harmans, and J.E. Mooij, Nature (London) 431, 159 (2004).

14. A. Wallraff, D.I. Schuster, A. Blais, L. Frunzio, R.-S. Huang, J. Majer, S. Kumar, S.M. Girvin, and R.J. Schoelkopf, Nature (London) 431, 162 (2004).

15. D.I. Schuster, A.A. Houck, J.A. Schreier, A. Wallraff, J.M. Gambetta, A. Blais, L. Frunzio, J. Majer, B. Johnson, M.H. Devoret, S.M. Girvin, and R.J. Schoelkopf, Nature (London) 445, 515 (2007).

16. A. Izmalkov, M. Grajcar, E. Il'ichev, T. Wagner, H.-G. Meyer, A.Yu. Smirnov, M.H.S. Amin, A.M. van den Brink, and A.M. Zagoskin, Phys. Rev. Lett. 93, 037003 (2004).

17. M. Grajcar, A. Izmalkov, S.H.W. van der Ploeg, S. Linzen, T. Plecenik, T. Wagner, U. Hübner, E. Il'ichev, H.-G. Meyer, A.Y. Smirnov, P.J. Love, A.M. van den Brink, M.H.S. Amin, S. Uchaikin, and A.M. Zagoskin, Phys. Rev. Lett. 96, 047006 (2006).

18. W.D. Oliver, Y. Yu, J.C. Lee, K.K. Berggren, L.S. Levitov, and T.P. Orlando, Science 310, 1653 (2005).

19. M. Sillanpää, T. Lehtinen, A. Paila, Y. Makhlin, and P. Hakonen, Phys. Rev. Lett. 96,187002 (2006).

20. A. Izmalkov, S.H.W. van der Ploeg, S.N. Shevchenko, M. Grajcar, E. Il'ichev, U. Hübner, A.N. Omelyanchouk, and H.G. Meyer, Phys. Rev. Lett. 101, 017003 (2008).

21. C.M. Wilson, T. Duty, F. Persson, M. Sandberg, G. Johansson, and P. Delsing, Phys. Rev. Lett. 98, 257003 (2007).
22. J. Hauss, A. Fedorov, C. Hutter, A. Shnirman, and G.S. Schön, Phys. Rev. Lett. 100, 037003 (2008).

23. M. Grajcar, S.H.W. van der Ploeg, A. Izmalkov, E. Il'ichev, H.-G. Meyer, A. Fedorov, A. Shnirman, and G.S. Schön, Nat. Phys. 4, 612 (2008).

24. I. Martin, A. Shnirman, L. Tian, and P. Zoller, Phys. Rev. B 69, 125339 (2004).

25. M. Grajcar, S. Ashhab, J.R. Johansson, and F. Nori, Phys. Rev. B 78, 035406 (2008).

26. S.O. Valenzuela, W.D. Oliver, D.M. Berns, K.K. Berggren, L.S. Levitov, and T.P. Orlando, Science 314, 1589 (2006).

27. J.E. Mooij, T.P. Orlando, L. Levitov, L. Tian, C.H. van der Wal, and S. Lloyd, Science 285, 1036 (1999).

28. I. Chiorescu, Y. Nakamura, C.J.P.M. Harmans, and J.E. Mooij, Science 299, 1869 (2003).

29. D.J. Wineland, R.E. Drullinger, and F.L. Walls, Phys. Rev. Lett. 40, 1639 (1978).

30. W. Neuhauser, M. Hohenstatt, P. Toschek, and H. Dehmelt, Phys. Rev. Lett. 41, 233 (1978).

31. I. Marzoli, J.I. Cirac, R. Blatt, and P. Zoller, Phys. Rev. A 49, 2771 (1994).

32. C. Monroe, D.M. Meekhof, B.E. King, S.R. Jefferts, W.M. Itano, D.J. Wineland, and P. Gould, Phys. Rev. Lett. 75, 4011 (1995).

33. S.N. Shevchenko, S. Ashhab, and F. Nori, Phys. Rep. 492, 1 (2010).

34. A.V. Shytov, D.A. Ivanov, and M.V. Feigel'man, Eur. Phys. J. B 36, 263 (2003).

35. L.D. Landau, Phys. Z. Sowjetunion 2, 46 (1932); G. Zener, Proc. R. Soc. London, Ser. A 137, 696 (1932); E.C.G. Stückelberg, Helv. Phys. Acta 5, 369 (1932).

36. J. Ankerhold and H. Grabert, Phys. Rev. Lett. 91, 016803 (2003).

37. G. Ithier, E. Collin, P. Joyez, D. Vion, D. Esteve, J. Ankerhold, and H. Grabert, Phys. Rev. Lett. 94, 057004 (2005).

38. K. Saito, M. Wubs, S. Kohler, P. Hanggi, and Y. Kayanuma, Europhys. Lett. 76, 22 (2006).

39. K. Saito and Y. Kayanuma, Phys. Rev. B 70, 201304(R) (2004).

40. G.Z. Sun, X.D. Wen, B. Mao, J. Chen, Y. Yu, P.H. Wu, and S.Y. Han, Nat. Commun. 1, 51 (2010).

41. S.N. Shevchenko, A.N. Omelyanchouk, and E. Il'ichev, Fiz. Nizk. Temp. 38, 360 (2012) [Low Temp. Phys. 38, 283 (2012)].

42. D.M. Berns, M.S. Rudner, S.O. Valenzuela, K.K. Berggren, W.D. Oliver, L.S. Levitov, and T.P. Orlando, Nature (London) 455, 51 (2008).

43. G.Z. Sun, X.D. Wen, Y.W. Wang, S.H. Cong, J. Chen, L. Kang, W.W. Xu, Y. Yu, S.Y. Han, and P.H. Wu, Appl. Phys. Lett. 94, 102502 (2009).

44. Y.W. Wang, S.H. Cong, X.D. Wen, C. Pan, G.Z. Sun, J. Chen, L. Kang, W.W. Xu, Y. Yu, and P.H. Wu, Phys. Rev. B 81, 144505 (2010).

45. J. Bylander, M.S. Rudner, A.V. Shytov, S.O. Valenzuela, D.M. Berns, K.K. Berggren, L.S. Levitov, and W.D. Oliver, Phys. Rev. B 80, 220506 (2009). 
46. W.D. Oliver and S.O. Valenzuela, Quant. Info. Proc. 8, 261 (2009).

47. D.M. Berns, W.D. Oliver, S.O. Valenzuela, A.V. Shytov, K.K. Berggren, L.S. Levitov, and T.P. Orlando, Phys. Rev. Lett. 97, 150520 (2006).

48. L.J. Du, M.J. Wang, and Y. Yu, Phys. Rev. B 82, 045128 (2010).

49. L.J. Du and Y. Yu, Phys. Rev. B 82, 144524 (2010).

50. M.S. Rudner, A.V. Shytov, L.S. Levitov, D.M. Berns, W.D. Oliver, S.O. Valenzuela, and T.P. Orlando, Phys. Rev. Lett. 101, 190502 (2008).

51. X.D. Wen and Y. Yu, Phys. Rev. B 79, 094529 (2009).

52. L.J. Du and Y. Yu, http://arxiv.org/abs/1012.2911.

53. L.J. Du and Y. Yu, http://arxiv.org/abs/1012.2917.

54. As shown in Refs. 46 and 48, taking relaxation rates constant is a good approximation. In Ref. 52, we analyzed detailedly the validity of this approximation. Please refer to Ref. 52 for detail.

55. M.H.S. Amin and D.V. Averin, Phys. Rev. Lett. 100, 197001 (2008).
56. M.H.S. Amin and F. Brito, Phys. Rev. B 80, 214302 (2009).

57. Equation (6) can be also used in other crossover. When the decoherence is weak, the transition region is almost near the crossover.

58. T. Lanting, A.J. Berkley, B. Bumble, P. Bunyk, A. Fung, J. Johansson, A. Kaul, A. Kleinsasser, E. Ladizinsky, F. Maibaum, R. Harris, M.W. Johnson, E. Tolkacheva, and M.H.S. Amin, Phys. Rev. B 79, 060509 (2009).

59. A.O. Niskanen, Y. Nakamura, and J.P. Pekola, Phys. Rev. B 76, 174523 (2007).

60. J.Q. You, Y.X. Liu, and F. Nori, Phys. Rev. Lett. 100, 047001 (2008).

61. This is reasonable because larger frequency leads to shorter time staying in the transition region. Larger amplitude is then necessary to extend the time of being in the transition region over one cycle.

62. The large detuning assures even the state is driven to completely reach the transition region near $\Delta_{12}$, the effect of $\Delta_{03}$ is still negligible. 